\title{
Miscellen.
}

\section{Der verfluchte Feigenbaum.}

Im Markusevangelium (1 1, I 2 ff. 20. 21) wird erzählt:

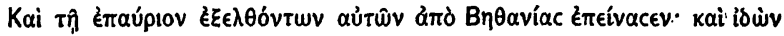

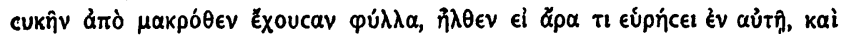

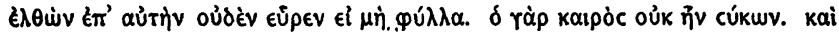

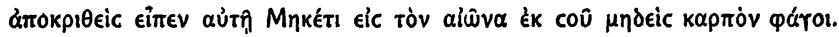

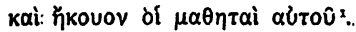

Am Abend kehrt Jesus mit den Jüngern von Teŗusalem nach Bethanien zurück, dann heißt es weiter:

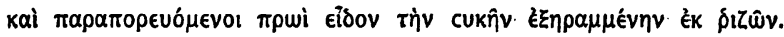

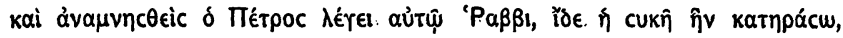
Ėñ́pavtal.

Jesus hat natülich gewußt, daßj.kurz vor dem Paschafest keine reifen Feigen an den Bäumen: $z u$ finden sind: wenn er trotzdem nach solchen sucht; hat er von dem Baum ein Wunder verlangt. Aber der Baum versagt; Jesus verflucht ihn und der Fluch geht in Erfüllung.

Lukas hat die Geschichte anstößig gefunden; er läßt sie fort. Matthaeus (2I, I8 ff.) lälst auf den.Fluch unmittelbar das Verdorren folgen, was den Ton des. Ganzen nicht verbessert. Ferner fehlt bei ihm der Hinweis darauf, daß.Jesus $z u$. jener Jahreszeit keine Feigen am Baume hätte finden können. Die Korrektur ist darum mißlungen, weil die Jahreszeit feststeht auch ohne jenen. Hinweis: und den Feigenbaum nach Galilaea $z \mathrm{u}$ verlegen, hat Matthaeus nicht gewagt. Andrerseits ist nicht zu leugnen, dab: die Geschichte ungemein gewinnt, wenn sie zur Zeit der reifen Feigen spielt.

I In $\mathrm{D}$ ist eine Umformung des Textes erhalten: kai $\tau$ दी Emaúptov $E E \in \lambda \theta \delta v \tau \alpha$ (aus

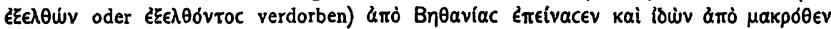

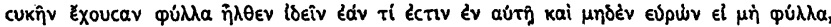

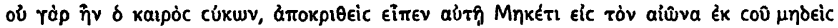

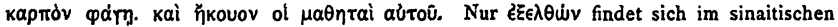
Syrer [مب saled wied; es ist Ausgleichung mit Mt 2I, 18: alle anderen Abweichungen in $D$ sind Versuche, den Stila zu verbessern. 
Hat also Matthaeus die Zeitangabe mit Recht entfernt oder nicht vorgefunden, so ist die Geschichte entweder überhaupt zeitlos oder eine dunkle Erinnerung daran, dab Jesus früher nach Bethanien und Jerusalem gekommen ist als es die auf die Katastrophe hinstrebende Tradition der Synoptiker darstellt (vgl. Wellhausen, Evang. Marci 94): die erste Möglichkeit ist mir wahrscheinlicher. Sei dem wie ihm sei, die Geschichte selbst klingt ganz naiv, wie ein Märchen, widerstrebt hartnäckig ethischer oder gar christologischer Exegese. Es scheint aber, als sei sie unvollständig: denn der Feigenbaum, der am Wege zwischen Bethanien und Jerusalem stand, kommt noch einmal im Evangelium vor.

Hinter der eschatologischen Rede Mk 13,4-27 stehen eine Reihe von abgerissenen, trümmerhaften Sprüchen, die nur verständlich werden, wenn man sie von einander isoliert und jeden für sich interpretiert; vgl. Wellhausen II 3 f. Der erste dieser Sprüche (I 3, 28f.) lautet:

'A

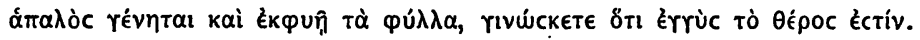

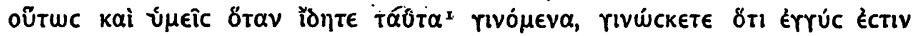
Ł̇ì $\theta$ úpalc.

Wer diese Worte exakt zu deuten versucht, stößt auf mehr als eine Schwierigkeit. Um des kà ú úîc willen verlangt Wellhausen für das erste rıvúckete die Aussprache rıvúcketal. Mish macht nicht so sehr bedenklich, daß die alten Übersetzungen auf rıvúcкєtє führen, die Verwechslung von $\epsilon$ und al kann sehr hoch hinaufreichen: aber das allgemeine passivische rıvúcketat ist sehṛ auffallend für ợ̂hóv éctıv, $\varphi \alpha-$

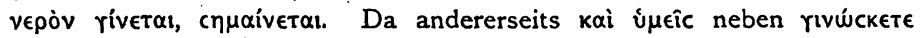
unerträglich ist, so wird sich kaum leugnen lassen, dab die Stelle gestört ist.

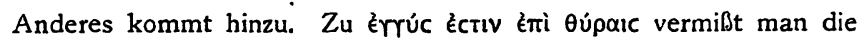
unzweideutige Bezeichnung des Subjekts' ${ }^{2}$; auch ist die Verkoppelung des eigentlichen Ausdrucks für die Nähe mit dem bildlichen um so unangenehmer als im Vergleich nur steht ätı Érrùc tò tépoc écrív. Der sinaitische Syrer paraphrasiert hier und Mt 24, 33 sehr geschickt

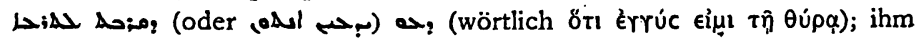
kam zu statten, daß er Érrùc cival mit einem -Wurt ausdrücken konnte.

I $\pi \alpha \dot{v \tau \alpha} \tau a 0 \tau \alpha \cdot$ findet sich in „abendländischen“ Texten und einigen Handschriften der Peschitta; die ältere syrische und die griechische Überlieferung hat nur $\tau \alpha \hat{\tau} \tau \alpha$. Wichtige Varianten zu den beiden Versen sind mir nicht bekannt.

2 Die Peschitta setzt das allgemeine Femininum va als wôrtliche Übersetzung korrekt, dem Sinne nach unverstắndlich: 


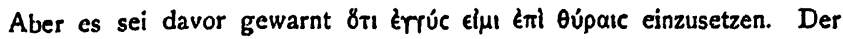

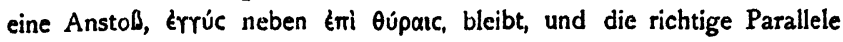

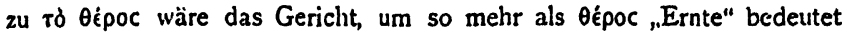
und die Einte mit allem was dazu gehört, das stehende neutestament-

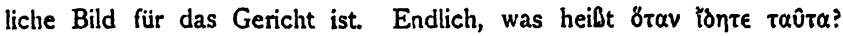
Das kann nur auf das gehn, was eben gesagt ist: aber da stehen nicht nur Zeichen, sondern das Ende selbst (13, 26. 27).

Matthaeus hilft nicht weiter (24, $32 \mathrm{ff}$ ). Lukas korrigiert, beweist also die Berechtigung der Anstöße, indem er die Stelle so umformt (21, 29ff.):

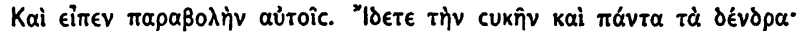

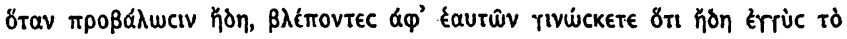

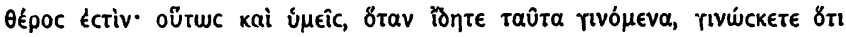

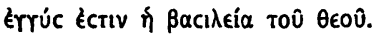

Durch die Einschiebung des Reiches Gottes wird allerdings das Er-

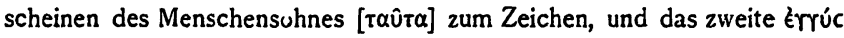
Éctıv - émi Oúpaıc ist gestrichen - erhält sein Subjekt: aber die präzise

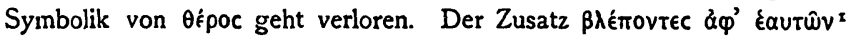

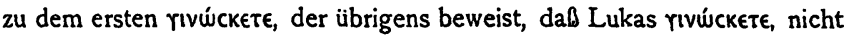

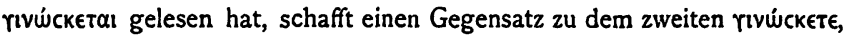
das nunmehr die übersinnliche Erkenñtnis der Zukunft bedeuten soll; k $\alpha \mathbf{i}$ bezieht sich nicht mehr auf ú $\mu$ eic, sondern auf den ganzen Satz und ist, wie oft im klassischen Griechisch, vom Personalpronomen nur attrahiert ${ }^{2}$. Das ist alles secundär, verrät den überlegt vorgehenden, die Mittel beherrschenden Stilisten.

Es sind bëi Lukas noch zwei weitere Anstöße weggeschafft, von denen bisher nicht die Rede gewesen ist. Aus der etwas unklaren Einführung bei Markus „Vom Feigenbaum aus lernet das Gleichnis verstehn“ - es geht keins vorher, und der Anschluß ans Vorhergehende ist weniger als notdürftig - wird bei Lukas das formelhafte „Und er sagte ihnen ein Gleichnis". Neben den einen Feigenbaum treten ,alle Bäume“, eine unsäglich matte Rede, gewiß; indeß was soll der eine, determinierte

× Er fehlt in D und den beiden alten Syrern durch Angleichung an die Paralleltexte bei MIt und Mk; ¿a' ' Eautŵv lesen die Peschitta und die Lateiner.

2 Der Sprachgebrauch wird oft.verkannt; ich gebe daher ein paar Belegstellen.

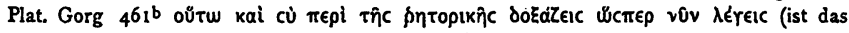

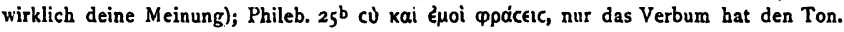

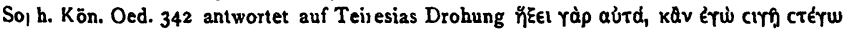

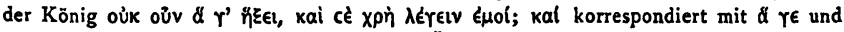

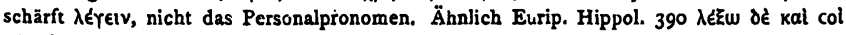

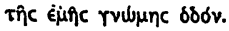


Feigenbaum in der allgemein giltigen Parabel? Auf Wellhausens Frage zu Mk 13, 28 „warum nicht, von den Bäumen“ überhaupt?" dürfte sich schwer eine Antwort finden lassen.

In den Worten Jesu bei Markus steckt ein Fremdkörper, der das Echte entstellt. Es schiebt sich alles zurecht, wenn man das zweite Glied des Vergleichs beseitigt, damit werden die Anstöße ausgerottet. Ich verbürge mich nicht dafür, daß der älteste Text jemals griechisch vorgelegen hat; dem Sinne nach muß der Spruch etwa gelautet haben:

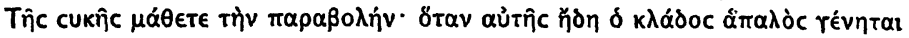

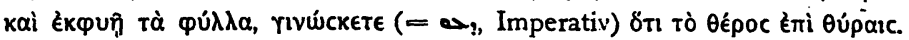

Der Feigenbaum, dessen Gleichnis verstanden werden soll, muß ein bestimmter sein, den alle kennen, eben der, den der Herr verflucht hat. Gibt man das zu, so muß man freilich, um die Verbindung zwischen den beiden Erwähnungen des merkwürdigen Baumes herzustellen, auch das Wort II, I4 für retouchiert erklären. Die alte Fassung; auch diese natürlich nur dem Sinne nach, ergibt sich aus der Deutung von 13, 28:

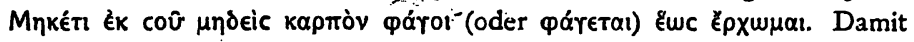
nun aber die uralte, schon sehr früh nicht mehr verstandene Erzählung zu einem klaren Ganzen zusammenkrystallisiert, ist ein realer Kernı nötig: das ist eben jener verdorrte Feigenbaum, der wirklich in der Zeit der Urgemeinde am Wege von Bethanien nach. Jerusalem gestanden haben muß. Die Geschichte, die von ihm erzählt wurde, ist das was die romantische, oder wie man gewöhnlich. sagt, die alexandrinische: Poesie der Hellenen ein aľrov nannte. Aľriov ist jede Erzählung, die etwas Reales, Gegenwärtiges, aber Unverständliches durch eine Geschichte mutiviert, sei. es ein seltsamer Brauch, eine nicht $2 \mathbf{u}$ deutende Zeremonie, ein survival wie man jetzt sagt, oder auch eine Ruine, von der es keine hist ırische Kunde gibt, oder eine auffallende Lokalität, ein sonderbar geformter Fels, ein alleinstehender; alter Baum, wie er die Phantasie der Orientalen leicht in Bewegung setzt; denn wenn auch die griechische Romantik, welche solche Geschichten' sammelte, verwertete und erfand, den präzisen Ausdruck dafür geprägt hat, die Sache findet sich überall.

Bethanien war ein uralter, wenn nicht der älteste Sammelpunkt solcher; die „auf das Reich Gottes warteten“. Wenn sie murgens. nach der heiligen Stadt gingen und abends heimkehrten; kamen. sie an dem verdorrten Feigenbaum vorbei, der, ein einsames Wahrzeichen, weithin. sichtbar, am Wege emporragte. Warum war er verdorrt? Als der Herr, so erzählten sie sich, zum ersten Male von Bethanien nach Jerusalem wanderte, da hungerte ihn, und er ging an den.Baum,-um sich.an seinen. 
Früchten zu erquicken. Der Baum gab keine her. Und der Herr sprach den Fluch uibcr ihn: „Keiner soll eine Frucht von dir essen, bis ich komme". Als an Tage danach der Herr mit den Jüngern wieder des Weges kam, war der Baum verd, rrt bis in die Wurzel; der Herr aber sprach zu den Jüngern: „Wenn der Baum wieder ausschlägt, ist die Ernte vor der Tür". Und die Gläıbigen sahen jedesmal, wenn sie vorbeikamen, hin, ob sich noch keine Knospe zeige.

Im altesten Evangelium wirds mehr von solcher auf freiem Felde gewachsenen Puesie gegeben haben; aber die Zeit kam nur zu früh, dab keiner sich fand, der das Heidekraut pflückte und aufhob.

Eduard Schwartz.

\section{Die Entstehung der Zahl 666.}

I.

Aus dem, was Corssen in dieser Zeitschr. 1903, 264 ff. wider mich geltend gemacht hat, ist mir nicht klar geworden, inwiefern ich ihn mißverstanden haben soll. Jedenfalls aber gibt er meine Meinung nicht richtig wieder, wenn er schreibt: „Denn darauf kommt es ihm (Vischer) an: es handelt sich hier um keinen Namen, sondern die Zahl 666 muß hier gedeutet werden, wie Irenaeus es tat, nämlich auf die ganze Summe der Ungöttlichkeit von Anbeginn der Welt an". Wenn dies wirklich meine Ansicht wäre, so hätte ich in der Tat die betreffende Stelle gründlich mißverstanden. Ich bin deshalb genötigt, so unbedeutend die Sache an sich ist, in aller Kürze noch einmal festzustellen, was ich zu erwägen gegeben habe.

Ich habe so wenig als Irenaeus bestritten, dab die Zahl 666 nach der Meinung des Apokalyptikers die Summe der Buchstaben eines Namens

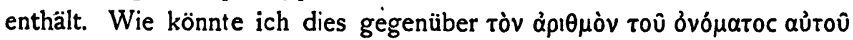

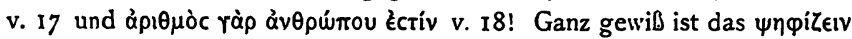
der Zahl, zu dem der Apokalyptiker auffordert, nicht Selbstzweck. Die Zahl des Tieres soll vielmehr berechnet werden, damit ein Zeichen gewonnen wird, an dem die kommende gottfeindliche Größe erkannt werden kann. Sie ist daran zu erkennen, da@ die Zahl, welche die Buchstaben ihres Namens ergeben, 666 ist. Deshalb habe ich auch nicht gesagt, die Zahl 666 müsse gedeutet werden, wie Irenaeus es tat.

IIch habe vielmehr die Frage einer erneuten Untersuchung unterzogen, wie die Apokalypse zu der Zahl 666 gekommen sei. 